\title{
Vitrectomy alone or Vitrectomy Combined with Scleral Buckle in the Management of Giant Retinal Tears
}

\author{
Ang Xiao ${ }^{1}$, Hui Feng Zhong ${ }^{2}$, Qi Ying Liu $^{1}$, Yi Shao ${ }^{1}$ and Qiong Zhou ${ }^{1}$ \\ ${ }^{1}$ Department of Ophthalmology, The First Affiliated Hospital of Nanchang University, China \\ ${ }^{2}$ Department of Intensive Care, The First Affiliated Hospital of Gannan Medical University, China
}

\begin{abstract}
The aim of this study was to compare as to which treatment achieves better outcomes in the management of giant retinal tears (GRTs) - pars plana vitrectomy (PPV) alone or combined with scleral buckle (SB)? The Web of Science, PubMed, and Cochrane Library databases were searched from January 1, 1950 to October 1, 2020. Pooled odds ratios (ORs), 95\% confidence intervals $(\mathrm{Cls})$, heterogeneity, and publication bias were determined with Review Manager software. PPV combined with SB significantly decreased the risk of recurrent retinal detachment ( $R R D, O R=0.39,95 \% \mathrm{Cl}=0.20-0.77, I^{2}=35 \%, \mathrm{p}=0.006$ ) in GRT management compared with PPV alone. However, the final anatomical success ( $\left.\mathrm{OR}=0.74,95 \% \mathrm{Cl}=0.23-2.39, l^{2}=0 \%, \mathrm{p}=0.61\right)$, final visual acuity $(\mathrm{OR}=1.11,95 \% \mathrm{Cl}=0.48-2.58, \mathrm{I} 2=13 \%, \mathrm{p}=0.81)$, and risk factors of $\mathrm{GRT} \geq 180^{\circ}(\mathrm{OR}=0.43,95 \% \mathrm{Cl}=$ $0.15-1.22, I^{2}=0 \%, p=0.11$ ) were not significantly different between the two approaches.

According to the final anatomical success, final visual acuity, and risk factors of GRT $\geq 180^{\circ}$, there were no significant differences between PPV combined with SB and PPV alone for the management of GRT in the current study, except in decreasing the risk of RRD.
\end{abstract}

Key Words: Giant retinal tear, Pars plana vitrectomy, Scleral buckling, Recurrent retinal detachment.

How to cite this article: Xiao A, Zhong HF, Liu QY, Shao Y, Zhou Q. Vitrectomy alone or Vitrectomy Combined with Scleral Buckle in the Management of Giant Retinal Tears. J Coll Physicians Surg Pak 2021; 31(08):953-958.

\section{INTRODUCTION}

The standard management of giant retinal tears (GRTs) has posed a great challenge to vitreoretinal ophthalmologists. Rarely, GRTs are due to giant rhegmatogenous retinal detachment, and these patients may have a poor prognosis. Rapidly developing and usually extensive retinal detachments commonly occurred with GRTs. One study estimated that the incidence of GRT is $0.05 / 100,000$ among the general population per year or $0.5-8.3 \%$ of all patients with rhegmatogenous retinal detachment, ${ }^{1}$ according to retrospective, single centre, hospital-based study reported, when it is the case with regard to other characteristics of harboring GRTs. There are many risk factors involved in GRT, such as Marfansyndrome, high myopia and trauma. ${ }^{2}$

Pars plana vitrectomy (PPV) was initially adopted as an adjunctive surgical step for GRT management and was subsequently accepted as the principle manoeuvre, in combination with a high posterior encircling buckle to prevent re-detachment due to anterior proliferative vitreoretinopathy (PVR).

Correspondence to: Qiong Zhou, Department of Ophthalmology, The First Affiliated Hospital of Nanchang University, China

E-mail: qiongz-ms@126.com

Received: March 26, 2021; Revised: May 29, 2021;

Accepted: June 19, 2021

DOI: https://doi.org/10.29271/jcpsp.2021.08.953
Due to a higher success rate than previously used surgical treatments, PPV has become the standard first-line treatment of $\mathrm{GRT},{ }^{3}$ but some surgeons prefer to perform an encircling scleral buckle or a scleral buckle for a portion of the circumference. To prevent concomitant scleral buckling (SB), some researchers suggested that an encircling buckle may provide vitreous base support, particularly in cases with anterior PVR. ${ }^{2}$ However, one groupdemonstrated thatencircling buckles did notprovideadditional benefit for patients GRTs; but instead resulted in potential complications such as tear fishmouthing, choroidal haemorrhage, and anterior retinal folds, facilitating tear slippage. ${ }^{4}$ Despite advances in surgical techniques and endotamponade agents, GRTs pose significant challenge due to their complex anatomy, the risk of retinal slippage, and a high incidence of PVR. The use of an SB combined with PPV for GRT management has been a point of contention.

The aim of this meta-analysis was to identify as to which approach would be to treat GRTs-PPV alone or PPV combined with SB.

\section{METHODOLOGY}

Ethical approval and patient consent were not required, since all analyses were based on previously published studies. The systemic literature review and meta-analysis were performed following the preferred reporting items for systematic reviews and meta-analyses (PRISMA) statement. ${ }^{5}$ 
A systematic search was performed on the Web of Science, PubMed and Cochrane Library databases from January 1, 1950 to October 1, 2020. Articles written in English, French, German, Japanese, and Korean were included. Various combinations of the terms giant retinal tear (giant retinal tears), GRT, and giant rhegmatogenous retinal detachment (giant rhegmatogenous retinal detachments)' were fed into the search engines. References cited in the identified articles were also reviewed to find related studies.

Two independent reviewers (HFZ and QYL) judged the articles on the principle of fairness and justice. Discrepancies were resolved through the discussion with a third-party reviewer (QZ) to avoid bias. To confirm some detailed data in the selection studies, a final confirmation was obtained by asking all authors to contribute any related information and statistics. The reference lists from original and review articles were also manually reviewed to identify appropriate studies. Articles were assessed for quality to determine inclusion in the meta-analysis after initially screening the titles and abstracts.

To eliminate the interference from other factors, studies consistent with the following criteria were included: (1) subjects ages ranging from 3 to 83 years; (2) study samples $\geq 15$; (3) large-circumferential retinal tears measuring $\geqq 90^{\circ}$, or $\geqq 3$ contiguous clock hours (4) surgical techniques including PPV and PPV+SB with other procedures such as perfluorocarbon liquids (PFCLs) and silicone oil; (5) GRT with or without PVR; (6) comparison of PPV alone or with SB; and (7) at least two of the following experimental results of anatomical success, final visual acuity, and recurrent retinal detachment (RRD). Studies that did not meet these inclusion criteria were excluded during the initial or final review.

Data were extracted by two independent reviewers (HFZ and QYL) from all eligible studies. Another reviewer (QZ) solved any divergent issues by consulting with the respective authors on original manuscripts. The following data are shown in the table for each included article: first author name, publication year, gender, age (mean $\pm \mathrm{SD}$ ), mean follow-up time, number of GRT, surgical aspect, anatomical success, final visual acuity, RRD, and risk factors.

Odds ratios (ORs) and 95\% confidence intervals ( $\mathrm{Cls}$ ) were calculated to estimate the outcomes. ${ }^{6}$ Due to the low or high levels of heterogeneity in different studies, a fixed-effects or randomeffects model was used for analyses. $I^{2}$ statistics were used to evaluate the degree of heterogeneity; $25 \%, 50 \%$ and $75 \%$ wereconsidered as low, moderate, and high scores, respectively. ${ }^{7}$ To assess the outcomes of the two different procedures (PPV or PPV combined with SB) to treat GRT, a subgroup analysis was performed to investigate the sensitivities of and relationship between the two treatment methods. Using summary estimates from the individual studies with a random-effect models, ${ }^{8}$ study results were statistically analysed and displayed as forest plots. Funnel plotswere generated to assess potential publication bias. ${ }^{9}$ Probability values $<0.05$ were defined as statistically significant. All data analyses were performed with Review Manager software, version 5.3.

\section{RESULTS}

The electronic search identified 1,272 studies, and 1,218 (95.8\%) articles were excluded through the abstract and title review. For the remaining 54 papers, 49 did not meet the criteria, and five manuscripts ${ }^{10-14}$ were eventually chosen for this analysis (Figure 1). The characteristics of these studies are demonstrated in Table I. The analysis comprised 256 eyes from five studies. Among these individuals, all the patients underwent PPV, including 169 eyes combined with SB and others without SB. Among the five included studies, three included almost all data, while two studies contained several important outcomes such as final visual acuity and RRD. The evaluation of outcomes showed results associated with PPV combined with SB and PPV alone, but the statistical methods, descriptions, and analyses of all studies varied and focused on differentaspects.

Five studies were identified, which included 256 eyes to compare the effects of PPV with or without SB in GRT management. ${ }^{10-14}$ Although several studies did not report baseline characteristics, the main information of the articles was used. Since low heterogeneity was observed between the studies $\left(I^{2}=\right.$ $35 \%$ ), a fixed-effect model was applied. Compared with PPV alone, PPV with SB significantly decreased the risk of RRD (OR $\left.=0.39,95 \% \mathrm{Cl}=0.20-0.77, l^{2}=35 \%, p=0.006\right)$ in GRT management (Figure 2). However, the final anatomical success $\left(O R=0.74,95 \% \mathrm{Cl}=0.23-2.39, I^{2}=0 \%, p=0.61\right)$ in four studies, ${ }^{10,12-14}$; final visual acuity $(\mathrm{OR}=1.11,95 \% \mathrm{Cl}=$ $0.48-2.58, I^{2}=13 \%, \mathrm{P}=0.81$ ) of three studies, ${ }^{10,12-14}$ and risk factors of $\mathrm{GRT} \geq 180^{\circ}\left(\mathrm{OR}=0.43,95 \% \mathrm{Cl}=0.15-1.22, I^{2}=0 \%, \mathrm{p}\right.$ $=0.11$ ) of three studies, ${ }^{10,12,14}$ revealed no significant differences between the two groups. In three studies, gender ( $Z=$ $1.15, p=0.25)$, age $(Z=0.63, p=0.53)$, the number of GRT $\left(<150^{\circ} / \geq 150^{\circ}\right)(Z=0.98, p=0.33)$, and mean follow-up time ( $Z=0.90, p=0.37$ ) were also not significantly different between the two groups in three studies. ${ }^{10,12,14}$

One study was excluded from the meta-analysis, owing to the small sample size for sensibility analysis. ${ }^{14}$ However, analysis of the otherfourstudies, ${ }^{10-13}$ revealed that PPV with SB was associated with a beneficial outcome $(O R=0.36,95 \% \mathrm{Cl}=$ $\left.0.18-0.72, I^{2}=44 \%, p=0.004\right)$, which is almost consistent with the analysis using all five studies, ${ }^{10-14}$ where PPV with SB decreased the risk of RRD in GRT management (Figure 3). However, there was no difference in final anatomical success (OR $=0.75,95 \% \mathrm{Cl}=0.21-2.71, I^{2}=0 \%, \mathrm{p}=0.44$ ) or final visual acuity $\left(\mathrm{OR}=1.03,95 \% \mathrm{Cl}=0.27-3.93, l^{2}=55 \%, p=0.96\right)$ between the two groups (Figures 4,5).

Despite the limited number of studies, they had low heterogeneity, and the funnel plot asymmetry suggested that there was no significant publication bias among the studies in the management of GRT comparing PPV+SB and PPV alone (Figure 6). 
Table I: Population characteristics of 5 GRT studies included in the meta-analysis.

\begin{tabular}{|c|c|c|c|c|c|c|c|}
\hline & $\begin{array}{l}\text { Gender } \\
\text { (No M/F) }\end{array}$ & Age (mean \pm SD) (years) & Mean follow-up time $(M \pm S D)$ & \multicolumn{2}{|c|}{$\begin{array}{l}\text { GRT degrees } \\
\left(\text { No }<150^{\circ} / \geq 150^{\circ}\right)\end{array}$} & \multicolumn{2}{|c|}{ Surgical Aspect (No) } \\
\hline & VT $\quad$ VT+SB & VT+SB & VT+SB & VT & VT+SB & & $\mathrm{VT}+\mathrm{SB}$ \\
\hline Kumar et al. $(2018)^{14}$ & $9 / 1 \quad 7 / 0$ & $35.00 \pm 22.63 \quad 14.71 \pm 9.60$ & $10.60 \pm 2.80 \quad 9.57 \pm 2.99$ & $5 / 5$ & $3 / 4$ & 10 & 7 \\
\hline Goezinne et al. $(2008)^{12}$ & $6 / 3 \quad 20 / 1$ & $56.33 \pm 13.07 \quad 51.90 \pm 10.13$ & $46.89 \pm 30.27 \quad 49.95 \pm 26.26$ & $7 / 2$ & $17 / 4$ & 9 & 21 \\
\hline Pitcher et al. (2015) & NA & NA & NA & NA & & 28 & 30 \\
\hline Verstraeten et al. (1995) & $18 / 2 \quad 12 / 2$ & $33.65 \pm 16.63 \quad 42.07 \pm 17.15$ & $18.55 \pm 14.80 \quad 15.71 \pm 6.81$ & $5 / 15$ & $7 / 7$ & 20 & 14 \\
\hline Al-Khairi et al. $(2008)^{11}$ & NA & NA & NA & NA & & 20 & 97 \\
\hline
\end{tabular}

\begin{tabular}{|c|c|c|c|c|c|c|c|c|}
\hline & $\begin{array}{l}\text { Anatomical } \\
\text { Success (\%) }\end{array}$ & $\begin{array}{l}\text { FVA } \\
\text { (No }\end{array}$ & //[0.1) & RRD ( & & Risk factors & & \\
\hline & VT VT+SB & VT & $\mathrm{VT}+\mathrm{SB}$ & VT & $\mathrm{VT}+\mathrm{SB}$ & HM & PVR & GRT $\geq 180^{\circ}$ \\
\hline & & & & $\mathrm{V} 1$ & $V I+S B$ & VT $\quad$ VT+SB & PVK & VT $\quad$ VT+SB \\
\hline Kumar et al. (2018) & $90 \% \quad 85.7 \%$ & $4 / 6$ & $3 / 4$ & $10 \%$ & $14.3 \%$ & $3 / 10 \quad 3 / 7$ & $17 \square 100 \% \square$ & $5 / 10 \quad 2 / 7$ \\
\hline Goezinne et al. (2008) & $100 \% 95.2 \%$ & NA & & $66.7 \%$ & $14.3 \%$ & NA & All A or B $(100 \%)$ & $3 / 21$ \\
\hline Pitcher et al.(2015) & $89.3 \% \quad 86.7 \%$ & NA & & $10.7 \%$ & $13.3 \%$ & NA & $C(17 \%)$ & NA \\
\hline Verstraeten et al. (1995) & $100 \% 100 \%$ & $4 / 16$ & $5 / 9$ & $45 \%$ & $14.3 \%$ & $8 / 20 \quad 7 / 14$ & NA & $10 / 20 \quad 3 / 14$ \\
\hline Al-Khairi et al. (2008) & NA & $12 / 8$ & $44 / 53$ & $35 \%$ & $18.60 \%$ & NA & $0(0 \%)$ & NA \\
\hline
\end{tabular}

M/F: Male/Female; SD: Standard deviation; VT: Vitrectomy; No: Number of.; FVA: Final visual acuity; RRD: Recurrent retinal detachment; HM: High myopia; NA: Not available.

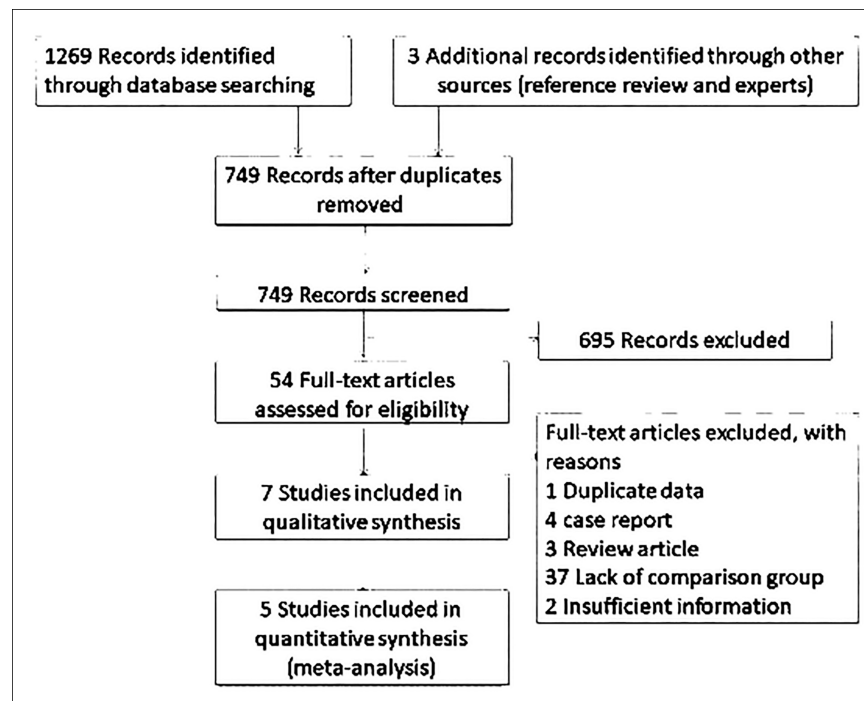

Figure 1: Flow diagram of the literature search according to the preferred reporting items for systematic reviews and meta-analyses (PRISMA) guidelines.

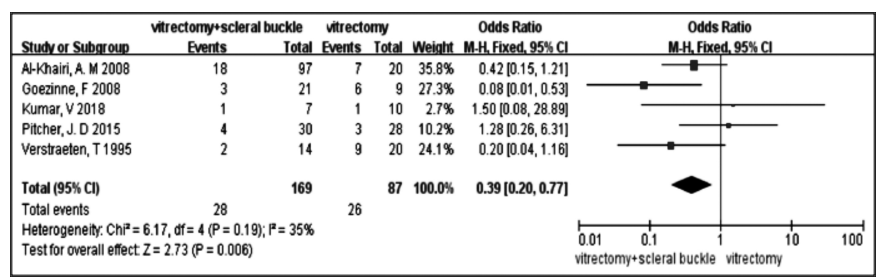

Figure 2: RRD following PPV + SB and PPV alone in the treatment of GRT.

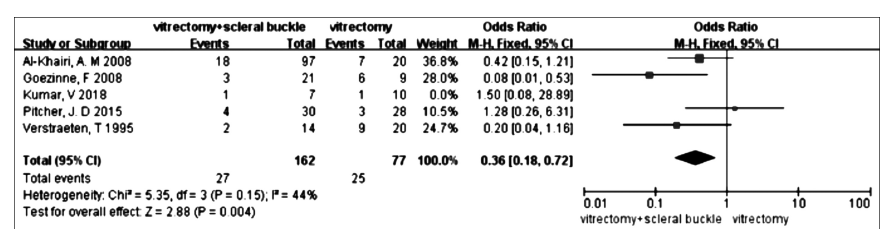

Figure 3: Sensibility analysis of RRD between PPV+SB and PPV alone in the treatment of GRT.

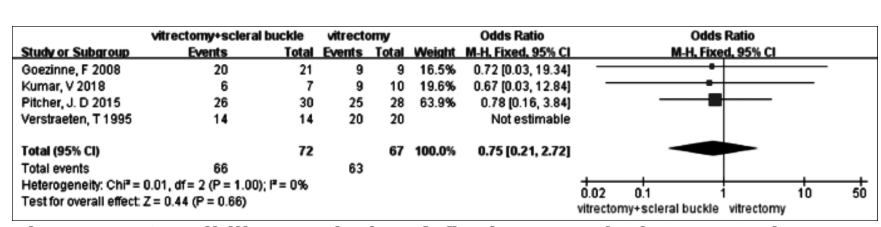

Figure 4: Sensibility analysis of final anatomical success between PPV+SB and PPV alone in the treatment of GRT.

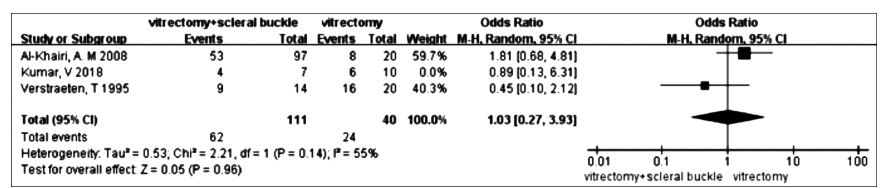

Figure 5: Sensibility analysis of final visual acuity between PPV+SB and PPV alone in the treatment of GRT.

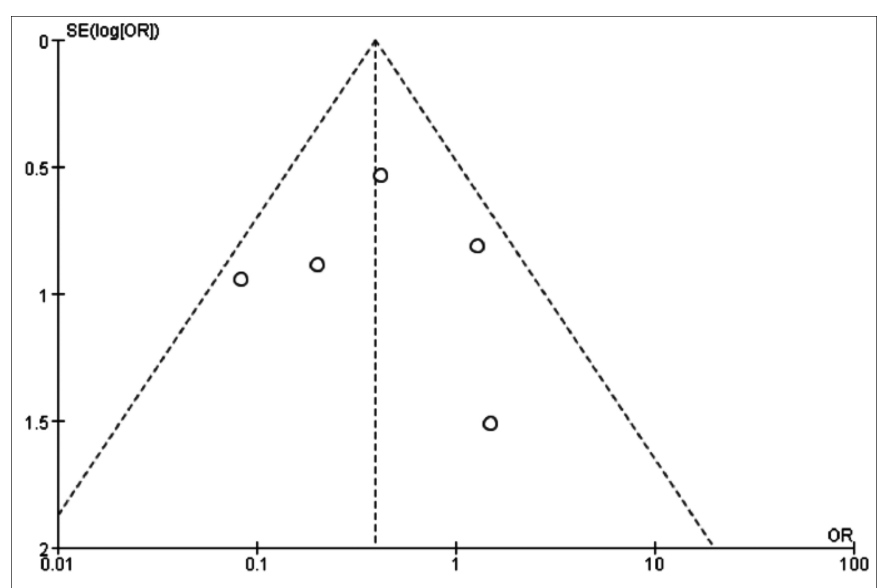

Figure 6: Funnel plot of studies comparing PPV+SB and PPV alone.

\section{DISCUSSION}

Effective treatment of GRT (a circumferential tear $\geq 90^{\circ}$ ), is very important for patient visual quality of life and is a great challenge to ophthalmologists due to technical treatment difficulties and the high risk of surgical complications. The incidence of GRT in the general United Kingdom population (0.09-0.11 per 100,000$)$ indicates that it may occur more commonly than previously thought $\left(0.05 / 100000\right.$ yearly). ${ }^{15}$ GRT carries a variety of risk factors: A posterior radial exten- 
sion at the ends of the tear, an inverted retinal flap, and high rates of PVR and foveal detachment. ${ }^{16-18}$ These complex cases may be technically difficult to manage surgically. However, the prognosis of GRT can be poor if patients are not promptly treated. ${ }^{19}$

Although GRTs pose several surgical challenges including flap manipulation, extensive PVR membranes, tear extension, flap slippage, and subretinal posterior PFCL or silicone oil, their management has been substantially more successful with modern surgical techniques. SB and PPV are very suitable for the treatment of phakic and pseudophakic eyes in GRT cases, respectively. ${ }^{20}$ However, there is a greater proportion of RRD among eyes without concomitant $\mathrm{SB}$, and further surgery is required for these cases ( $45 \%$ RRD rate in PPV alone vs. $14.3 \%$ in PPV + SB). ${ }^{10}$ Goezinne et al. demonstrated that the absence of SB was a risk factor for RRD in GRT management, ${ }^{12}$ which is consistent with another study ${ }^{21}$ Another report concluded that encircling SB and silicone oil tamponade would decrease the risk of RRD. ${ }^{11}$ The current study indicated that the most common method of repairing GRT-induced retinal detachment is a combination of PPV/SB and PFCLs, which obtains reasonable and generally favourable anatomical and visual results. ${ }^{22}$ Conversely, two groups recommended meticulous vitreous base trimming with deep scleral indentations and demonstrated that the GRT (with an appropriate tamponade) in cases with no significant PVR could be treated without SB placement. $3,23,24$ Some authors reported that successful treatment of GRT with initial micro-incision vitrectomy surgery (MIVS) does not need to be combined with an encircling $\mathrm{SB}^{25,26}$ which was confirmed by other investigators. ${ }^{18,27} \mathrm{~A}$ high rate of anatomical success is proposed ${ }^{28}$ and improvement of final visual acuity $^{14}$ was achieved with PPV alone compared to PPV and SB. For surgeons, who routinely placed encircling buckles, the reoperation rate was $27 \%-50 \%$, compared to $6 \%-21 \%$ for those who never placed encircling buckles.

The use of an SB combined with PPV for managing GRTs has been a point of controversy among researchers. ${ }^{12,16,18}$ In this meta-analysis of five studies with 256 eyes, it was found that PPV combined with SB was better than PPV alone with regard to decreasing the risk of RRD, but there was no significant difference in gender, age distribution, the extent of GRT, mean follow-up time, final anatomical success, final visual acuity, and risk of GRT $\geq 180^{\circ}$ between groups. Surgical techniques and treatments are constantly improved in GRT management. Intraoperative PFCL application dramatically increases reattachment rates after GRT surgery. ${ }^{2}$ One study showed that the use of a "sandwich" of anterior silicone oil and PFCL was comfortable for patients undergoing laser surgery and created well-formed closed vitreous chamber that promoted continuous maintenance of retinal attachment in eyes with GRT. ${ }^{29}$ Additionally, smaller-gauge vitrectomy has evolved over time and has several advantages including a high rate of anatomical success, lesser vitreous traction, and good PVR management. ${ }^{30-32}$ Other recent technical advancements such as MIVS, wide viewing systems, and chandelier illumination have also greatly improved GRT treatment (primary reattachment rate $>90 \%) .{ }^{18,25,28}$ Zhang et al. also reported that the 27 -gauge PPV combined with temporary PFCL application could be safe and effective for GRT management. ${ }^{33}$

There are no precise guidelines regarding intraoperative procedures of PPV for GRT; for example whether to use lensectomy, PFCL application, and $360^{\circ}$ endophotocoagulation. Advanced PPV techniques have significantly increased the rate of anatomical success in GRT treatment. ${ }^{17}$ Patient age, gender, extent of GRT $\left(<150^{\circ} / \geq 150^{\circ}\right)$, final anatomical success, final visual acuity, risk factors of GRT $\geq 180^{\circ}$, and the mean follow-up time were not significantly different between the PPV alone and PPV + SB groups. Due to the larger sample size of one study, ${ }^{11}$ heterogeneity increased when that study ${ }^{14}$ was excluded. It should also be noted that a GRT-associated detachment should be treated as any other rhegmatogenous retinal detachment, and adjuvant SB should only be considered in certain high-risk cases. ${ }^{18,34}$ Consequently, SB was performed at the discretion of the surgeon based on the therapeutic strategy of GRTs, which is appropriate for patients with persistent retinal contraction, advanced-grade PVR, extensive inferior pathology within a detached area. Furthermore, SB could create an inward indentation in the sclera and choroid, which would promote retinal reattachment, weaken traction of the vitreous around the retinal tear, and possibly reduce the risk of RRD by pre-emptively neutralising subsequent traction. ${ }^{20}$ Placement of an SB prior to the vitrectomy may counterbalance vitreous base contraction and peripheral traction, which could also compensate for retinal surface shortening induced by membranes with PVR in complicated cases including GRT, ${ }^{18,28}$ by preventing liquid in the vitreous cavity from re-entering the subretinal cavity through pushing the pressurised substance to close the retinal tear and reduce the vitreous cavity. This promotes contact between the detached retinal neuroepithelial and pigmented epithelial layers. Therefore, PPV combined with SB could enhance retina reattachment and effectively eliminate or reduce the effect of peripheral forces on residual traction, which can significantly decrease the risk of RRD. However, we should be aware that the recurrence rate of GRTs is relatively low in the clinic. The addition of SB can increase pain and the risks factor of high intraocular pressure and refractive system abnormalities.

\section{CONCLUSION}

The current analysis found no difference between PPV with or without SB with regard to the degree of GRT, mean followup time, final anatomical success, final visual acuity, or risk factors of GRT $\geq 180^{\circ}$; however, the addition of SB did decrease the risk of RRD. It is hoped that these findings provide some guidance for the treatment of GRT and improve patient quality of life. 
More detailed comparisons and larger studies are needed to verify the findings of this study.

\section{DATA AVAILABILITY:}

The meta-analysis data, used to support the findings of this study, are available from the corresponding author upon request.

\section{CONFLICT OF INTEREST:}

The authors declared no conflict of interest.

\section{ACKNOWLEDGMENTS:}

We must thank Mr. Wen Deng for his technical assistance, and Mr. Yi Shao for editing and proofreading. The present study was supported by the National Natural Science Foundation of China (Grant No. 13007280).

\section{AUTHORS' CONTRIBUTION:}

$A X$ : Concept, design and writing - original draft.

$\mathrm{HZ}$ : Acquisition, analysis, or interpretation of data.

QL: Methodology, Software and Statistical analysis.

YS: Statistical analysis and writing review and editing.

QZ: Supervision and writing review and editing.

\section{REFERENCES}

1. Chou SC, Yang $\mathrm{CH}$, Lee $\mathrm{CH}$, Yang $\mathrm{CM}$, Ho TC, Huang JS, et al. Characteristics of primary rhegmatogenous retinal detachment in Taiwan. Eye (Lond) 2007; 21(8):1056-61. doi: 10.1038/sj.eye.6702397.

2. Lee SY, Ong SG, Wong DW, Ang CL. Giant retinal tear management: An Asian experience. Eye (Lond) 2009; 23(3):601-5. doi: 10.1038/eye.2008.48.

3. Ambresin A, Wolfensberger TJ, Bovey EH. Wolfensberger, Bovey EH. Management of giant retinal tears with vitrectomy, internal tamponade, and peripheral 360 degrees retinal photocoagulation. RETINA 2003; 23(5):622-8. doi: 10.1097/00006982-200310000-00003.

4. Dong HL, Kyong HK, Sung WP, IKSoo B, Ji-Eun L. Vitrectomy with perfluorocarbon liquid versus combined encircling for retinal detachment with giant retinal tear. J Korean Ophthalmological Society 2015; 56(12):1880-6.

5. Liberati A, Altman DG, Tetzlaff J, Mulrow C, Gøtzsche PC, Ioannidis JPA, et al. The PRISMA statement for reporting systematic reviews and meta-analyses of studies that evaluate healthcare interventions: Explanation and elaboration. BMJ 2009; 339:b2700. doi: 10.1136/bmj.b2700.

6. McNutt LA, Wu C, Xue X, Hafner JP. Estimating the relative risk in cohort studies and clinical trials of common outcomes. Am J Epidemiol 2003; 157(10):940-3. doi: 10.1093/aje/kwg074.

7. Higgins JP, Thompson SG, Deeks JJ, Altman DG. Measuring inconsistency in meta-analyses. BMJ 2003; 327(7414): 557-60. doi: 10.1136/bmj.327.7414.557.

8. Dersimonian R, Nan L. Meta-analysis in clinical trials. Contr Clin Trials 1986; 7(3):177. doi: 10.1016/j.cct.2015.09.002.

9. Begg C, Mazumdar M. Operating characteristics of a rank correlation test for publication bias. Biometrics 1995;
50(4):1088-1101. doi.org/10.2307/2533446.

10. Verstraeten T, Williams GA, Chang S, Cox MS Jr, Trese MT, Moussa $\mathrm{M}$, et al. Lens-sparing vitrectomy with perfluorocarbon liquid for the primary treatment of giant retinal tears. Ophthalmology 1995; 102(1):17-20. doi: 10.1016/ s0161-6420(95)31063-9.

11. Al-Khairi AM, Al-Kahtani E, Kangave D, Abu El-Asrar AM. Prognostic factors associated with outcomes after giant retinal tear management using perfluorocarbon liquids. Eur J Ophthalmol 2008; 18(2):270-7. doi: 10.1177/1120672 10801800216.

12. Goezinne F, LA Heij EC, Berendschot TT, Gast ST, Liem AT, Lundqvist IL et al. Low redetachment rate due to encircling scleral buckle in giant retinal tears treated with vitrectomy and silicone oil. RETINA 2008; 28(3):485-92. doi: 10.1097/IAE.0b013e318150d879.

13. Pitcher JD, Khan MA, Storey PP, Hsiao-Fang-Yen N, Dollin ML, Hsu J, et al. Contemporary management of rhegmatogenous retinal detachment due to giant retinal tears: A consecutive case series. Ophthalmic Surg Lasers Imaging Retina 2015; 46(5):566-70. doi: 10.3928/23258160-20150521-08.

14. Kumar V, Kumawat D, Bhari A, Chandra P. Twenty-fve-gauge pars plana vitrectomy in complex retinal detachments associated with giant retinal tear. Retina 2018; 38(4):670-7. doi: 10.1097//AE.0000000000001592.

15. Ang GS, Townend J, Lois N. Epidemiology of giant retinal tears in the United Kingdom: The british giant retinal tear epidemiology eye study (BGEES). Invest Ophthalmol Vis Sci 2010; 51(9): 4781-7. doi: 10.1167/iovs.09-5036.

16. Shunmugam M, Ang GS, Lois N. Giant retinal tears. Surv Ophthalmol 2014; 59(2): 192-216. doi: 10.1016/j.survophthal.2013.03.006.

17. Berrocal MH, Chenworth ML, Acaba LA. Chenworth, LA. Acaba. Management of giant retinal tear detachments. J Ophthalmic Vis Res 2017; 12(1): 93-7. doi: 10.4103/ 2008-322X.200158.

18. Hocaoglu M, Karacorlu M, Ersoz MG, Sayman Muslubas I, Arf S. Vitrectomy with silicone oil tamponade for retinal detachment associated with giant retinal tears: Favourable outcomes without adjuvant scleral buckling. Acta Ophthalmol 2019; 97(2): e271-e6. doi: 10. 4103/2008322X.200158.

19. Diagne JP, De Medeiros ME, Ka AM, Ndiaye JM, Sow AS, Wane AM, Ndoye Roth PA, et al. Giant retinal tears: Senegalese experience. J Fr Ophtalmol 2019; 42(2):133-7. doi: 10.1016/j.jfo.2018.06.006.

20. Kunikata H, Abe T, and Nakazawa T. Historical, current and future approaches to surgery for rhegmatogenous retinal detachment. Tohoku J Exp Med 2019; 248(3):159-68. doi: 10.1620/tjem.248.159.

21. Scott IU, Murray TG, Flynn Jr HW, Feuer WJ, Schiffman JC. Outcomes and complications associated with giant retinal tear management using perfluoro-n-octane. Ophthalmology 2002; 109(10):1828-1833. doi: 10.1016/s0161-6420(02) 01184-3.

22. Rodriguez M, Lin J, Townsend JH, Smiddy WE, Albini TA, Berrocal AM, et al. Giant retinal tears: Clinical features and outcomes of vitreoretinal surgery at a university teaching hospital (2011-2017). Clin Ophthalmol 2018; 12:2053-8. 
doi: 10.2147/OPTH.S180353.

23. Freeman HM, Castillejos ME. Castillejos. Current management of giant retinal breaks: Results with vitrectomy and total air fluid exchange in 95 cases. Trans Am Ophthalmol Soc 1981; 79: 89-102.

24. Kreiger $A E$, Lewis $H$. Management of giant retinal tears without scleral buckling. Use of radical dissection of the vitreous base and perfluoro-octane and intraocular tamponade. Ophthalmol 1992; 99(4):491-7. doi: 10.1016/s01616420(92)31942-6.

25. Kunikata H, Abe T, Nishida K. Successful outcomes of 25and 23-gauge vitrectomies for giant retinal tear detachments. Ophthalmic Surg Lasers Imaging 2011; 42(6):487-92. doi: 10.3928/15428877-20110901-01.

26. Kunikata $\mathrm{H}$. Management of giant retinal tears using microincision vitrectomy surgery. Dev Ophthalmol 2014; 54:182-7. doi: 10.1159/000360465.

27. Quezada-Ruiz C, Cano-Hidalgo RA. Cano-Hidalgo, Giant retinal tears treated with lens sparing, bimanual $23 \mathrm{~g}$ vitrectomy without scleral buckle. Cir Cir 2014; 82(3):245-51.

28. Tabandeh H, London NJS, David B, Harry WFJ. Outcomes of small-gauge vitreoretinal surgery without scleral-depressed shaving of the vitreous base in the era of wide-angle viewing systems. Br J Ophthalmol 2019; 103(12):1765-8. doi: 10.1136/bjophthalmol-2018-313626.

29. Madana Gopalan VG. Sandwich technique with anterior sili- cone oil and posterior perfluorocarbon liquid for intraoperative retinal stabilisation in eyes with large retinal breaks. J Ophthalmic Vis Res 2019; 14(2):232-5. doi: 10.4103/ jovr.jovr_225_17.

30. Oshima Y, Wakabayashi T, Sato T, Ohji M, Tano Y. A 27gauge instrument system for transconjunctival sutureless microincision vitrectomy surgery. Ophthalmol 2010; 117(1):93-102 e2. doi: 10.1016/j.ophtha.2009.06.043.

31. Khan MA, Shahlaee A, Toussaint B, Hsu J, Sivalingam A, Dugel PU, et al. Outcomes of 27 gauge microincision vitrectomy surgery for posterior segment disease. Am J Ophthalmol 2016; 161: 36-43 e1-2. doi: 10.1016/j.ajo.2015.09.024.

32. Rizzo S, Barca F, Toussaint B, Hsu J, Sivalingam A, Dugel PU, et al. Twenty-seven-gauge vitrectomy for various vitreoretinal diseases. RETINA 2015; 35(6):1273-8. doi: 10.1097/IAE.0000000000000545.

33. Zhang Z, Wei Y, Jiang X, Zhang S. Surgical outcomes of 27gauge pars plana vitrectomy with short-term postoperative tamponade of perfluorocarbon liquid for repair of giant retinal tears. Int Ophthalmol 2018; 38(4):1505-13. doi: 10.1007/s10792-017-0613-4.

34. Jain N, Kozak JA, Niziol LM, Musch DC, Zacks DN. Vitrectomy alone in the management of giant retinal tears. Ophthalmic Surg Lasers Imaging Retina 2014; 45(5): 421-7. doi: 10.3928/23258160-20140908-03. 